\title{
ALGUNOS DATOS SOBRE EL COMERCIO ENTRE AL-ANDALUS Y EL NORTE DE ÁFRICA EN LA ÉPOCA OMEYA (I): LOS PUERTOS DE CONTACTO*
}

Por

ENRIQUE GOZALBES CRAVIOTO

A lo largo de la Edad Media existieron múltiples lazos humanos, económicos y culturales entre las dos orillas del Occidente islámico. Este hecho es algo bien conocido al nivel de una generalidad que, en no pocas ocasiones, resulta rayana en lo tópico. La cercanía de los territorios y el hecho de poseer una zona y otra una civilización similar, son factores que influyeron decisivamente en estas relaciones. Es bien cierto que las conexiones entre ambas costas han sido mucho más destacadas en lo referido al territorio específico de Andalucía. Recientemente, en las páginas de esta misma revista, $M$. de Epalza ha destacado que la zona oriental peninsular, el Sharq al-Andalus, no se vio tampoco al margen de estas intensas relaciones norteafricanas (1).

Como hemos indicado, la importancia de los contactos con el Norte de África tiene un carácter tópico en la historiografía. No obstante, resulta paradójico que, a la hora de concretar la afirmación general, los datos específicos no resulten precisamente numerosos. En lo que ahora nos interesa, encontramos un vacío de estudios y una documentación aparentemente escasa en todo aquello referido al comercio entre al-Andalus y el Norte de África. Esta ausencia de estudios es particularmente sensible debido a la importancia que, a nuestro juicio, tiene la cuestión que nos ocupa. $Y$ este vacio de investigaciones se produce en un terreno en el cual un sector de las fuentes tiene referencias relativamente numerosas.

- Dada la longitud del trabajo se ha preferido el publicarlo dividido en dos partes. La segunda, dedicada a los productos del comercio, a la evolución del mismo y a las conclusiones, aparecerá en un șiguiente número de Sharg al-Andalus.

(1) M. DE EPALZA: "Costas alicantinas y costas magrebíes: el espacio marítimo musulmán según los textos árabes". Sharq al-Andalus, 3, 1986, pp. 25-31, donde utiliza como elemento fundamental la descripción de al-Bakri. 
Significativo es a este respecto la insuficiencia del análisis de Lévi-Provençal en su historia de la España Omeya (2). El análisis de Lévi-Provençal acerca del comercio exterior de al-Andalus fue de tipo muy general, no entró en mayores concreciones y no superó los tópicos al uso. Se limitó a señalar que el comercio exterior de al-Andalus, tanto de importación como de exportación, se fundamentó únicamente en artículos de lujo; ya en el siglo X Almería se había convertido en el puerto básico por donde salian Ifriqiya y el Oriente islámico, y por donde entraban los productos procedentes de Iraq y Bizancio en ruta hacia Córdoba (3). Después indicaba que «las cáfilas de bestias de carga recorrían las principales vias de comunicación, dirigiéndose a Córdoba o a las demás grandes ciudades o, por el contrario, a los puertos de exportación como Algeciras, Málaga y Almería» (4). Un esquema que resulta cierto pero que es insuficiente. Además, el análisis centra excesivamente el análisis en Córdoba y en los puertos andaluces. No podemos dejar de lado que la costa desde Tortosa a Murcia también participó en el espacio marítimo común con el Norte de África y con sus relaciones (5).

Junto a esta rectificación, necesaria de formular, en las conclusiones de LéviProvençal existen dos puntos importantes que consideramos discutibles. El primero de ellos es la primacía del comercio andalusí con el Oriente islámico con respecto al practicado con el Norte de África. El segundo punto cuestionable es la concreción a los artículos de lujo como los principales del comercio exterior de al-Andalus. Contestamos este esquema por cuanto, en el primer punto, no se han analizado detalladamente los datos aportados por los geógrafos contemporáneos. En el segundo punto porque la reconstrucción es puramente imaginativa pero sin una apoyatura documental.

Con el presente trabajo queremos profundizar en el conocimiento de una parte esencial del comercio exterior de al-Andalus: el realizado con los distintos territorios del Norte de África. Para este estudio resulta básico delimitar las líneas de navegación seguidas por el comercio marítimo. Al-Andalus era para los norteafricanos un territorio separado por el mar. Era también un territorio musulmán del cual le separaban algunos aspectos políticos y religiosos que la propia intervención, política y militar de los Omeyas no dejó de traslucir (6). Pero esas diferencias geográficas, el mar de por medio, ni las políticas o religiosas, fueron capaces de frenar el desarrollo del comercio. Este comercio entre al-Andalus y el Norte de África alcanzó ya un notable desarrollo en el siglo iX pero fue en la época califal cordobesa cuando alcanzó su

(2) E LEVVI-PROVENCAL: Esoaña musulmana hasta la caída del Califato de Córdoba (711-1031 de /.C.). Instituciones y vida social e intelectual. T. V de la Historia de España dirigida por R. MENÉNDEZ PIDAL, Madrid, 1957, pp. 182 y ss. En absoluto trata de la cuestión, pese al título, S.M. IMAUDDIN: Economic hisiory of Muslim Spain under the Umåyyads. Dacca, 1963. Algunos datos sobre los productos, referidos sobre todo al comercio oriental, en P. MARTÍNEZ MONTÁVEZ: Exportaciones de al-Andalus al mundo arabe en la Edad Media. Valencia, 1965

(3) E. LÉVI-PROVENÇAL, op. cit., p. 183. Sobre la importancia del puerto de Almería, E. MOLINA LÓPEZ: «AIgunas consideraciones sobre la vida socio-económica de Almería en el siglo XI y primera mitad del XIl». Actas del IV Coloquio Hispano-Tunecino, Madrid, 1983, pp. 187-188.

(4) E. LÉVI-PROVENÇAL, op. cit., p. 189.

(5) M. DE EPALZA, op. cit.

(6) J. VALLVÉ: «La intervención Omeya en el Norte de Africa». Cuadernos de la Biblioteca Española de Tetuán. 4, 1967, pp. 7-39. 
mayor nivel. Entonces se sentaron las bases de su fuerte continuidad en siglos sucesivos.

\section{La documentación de estudio}

La mejor fuente para el conocimiento del comercio entre al-Andalus y el Norte de África en los siglos IX y X son las referencias de los geógrafos árabes de la época. Muy especialmente, hay que destacar a los geógrafos orientales. Nos hallamos con una documentación muy poco utilizada, escasamente analizada y en no pocas ocasiones despreciada. Es bien cierto que en estos geógrafos orientales nos encontramos con descripciones de al-Andalus y del Magreb que son excesivamente fragmentarias y que sólo ofrecen un conocimiento parcial (7). Pero no es menos cierto que contienen algunos datos de cierta envergadura, variable en todo caso. No podemos negar que el principal valor de esta documentación es su carácter de espontaneidad, para el estudio de al-Andalus en los siglos IX y $X$ nos hallamos ante testimonios contemporáneos (8).

El error de partida en el análisis de Lévi-Provençal consistió precisamente en un cierto desprecio de estas informaciones. No obstante, son las únicas que permiten una aproximación al tema que nos ocupa tanto en la vertiente de los datos concretos como en las conclusiones generales. Por otra parte, los textos de estos geógrafos árabes deben de analizarse no de manera simplemente sectorial, no deben concretarse a las informaciones suministradas en las descripciones de al-Andalus. Cuando hablan de diversas ciudades o regiones norteafricanas incluyen algunos datos de interés acerca de la presencia en ellas de comerciantes procedentes de al-Andalus. Dado que estas son las fuentes que vamos a utilizar en el presente trabajo estimamos conveniente el hacer una breve presentación de las mismas.

El primero de estos autores orientales de obras geográficas es Ibn Jurdadbih. Oficial de correos de origen persa, hacia el año 844 escribió un libro acerca de los reinos y los distintos caminos existentes entre ellos. Fue así el creador de este tipo de obras que iban a tener, por su carácter práctico, un gran desarrollo en el mundo árabe. Su descripción de al-Andalus y del Norte de África es muy esquemática, incluso sus datos no son de la época sino de bastantes años anteriores (9).

El segundo de estos geógrafos es al-Yáqübi, escritor de origen iraquí que ha-

(7) E. LÉVI-PROVENÇAL, op. cit, pp. 131-133.

(8) J. ALEMANY BOLUFER: «La geografía de la Peninsula lbérica en los escritores árabes». Revista del Centro de Estudios Históricos de Granada y su Reino, 9, 1919, pp. 109-152. Un análisis más breve en G. CORNU: "Les géographes orientaux des IX et X siècles et al-Andalus". Sharq al-Andalus, 3, 1986, pp. 11-18. Sobre el ambiente cultural de los mismos, R. ARIE: «Al-Andalus vu par quelques lettrées orientaux au Moyen Age». Andalucia lslámica, 2-3, 1981-82, pp. 71-84.

(9) IBN JURDADBIH: Kitab al-masalik wa-tmamalik. Edición y trad. francesa de M.J. DE GOEGE, en la Biblioteca Geographorum Arabicorum (B.G.A.), t. IV, Leyden, 1889. Algunos párrafos sobre el Occidente encontramos traducidos en R. BLACHERE: Extraits des principaux géographes arabes du Moyen Age. Paris-Beyrouth, 1932, pp. 26 y ss.; M. HADJ-SADOK: Description du Maghreb et de /'Europe au $/ / I=1 X$ siècle, Argel, 1949 Una traducción española de la parte de al-Andalus en J. ALEMANY BOLUFER, op. cit., pp. 111-115. Sobre este autor, el artículo de M. HADJ-SADOK en L'Encyclopedie de I/S/am (E./.), 2. a ed., t. III, o. 863. Sobre todos estos geógrafos puede verse el magnífico estudio de A. MIQUEL: La géographie humaine du monde musuiman jusqu'au milieu du XI siècle. T. i, Paris, 1967 (2. ${ }^{a}$ ed., 1973). 
cia el año 889 dio fin a su obra dedicada a describir los distintos países musulmanes (10). El principal valor en lo que nos interesa es que Ya'qūbī recoge el testimonio de un viajero que cruzó desde el Norte de África a al-Andalus.

El tercer geógrafo es de una importancia menor. Nos referimos a lbn al-Faqih al Hamadāni, autor hacia el año 902 de otro libro de descripción de los paises (11). En lo referido al occidente su obra no viene a ser otra cosa que una especie de compendio de la Ibn Jurdadbih.

En el año 921 escribió otro libro dedicado a la descripción de los paises y a los distintos itinerarios el geógrafo al-Istajri. Su obra ya es mucho más extensa que las anteriores, ofreciéndonos datos bastante más novedosos (12).

La obra de al--Istajrı fue ampliada y completada por otro geógrafo oriental, Ibn Hawqal. En este caso nos encontramos con un testimonio excepcional, puesto que Ibn Hawkal visitó personalmente tanto al-Andalus como el Norte de África a mediados del siglo $X(13)$.

Finalmente, hacia el año 988 escribió su descripción de los países musulmanes un último autor que vamos a utilizar, el también oriental al-Muqaddası. Los datos que nos ofrece son bastante completos, aunque no visitara personalmente al-Andalus (14). Mención especial merecen sus referencias al comercio de esclavos en el Occidente.

Estos datos de los geógrafos orientales, hemos mencionado los únicos que ofrecen datos para nuestro estudio, pueden y deben completarse con otras tres obras clasificables como de autores andalusís y occidentales. La primera de ellas es la de Abi-l-Husayn al-Zayyät, autor andalusí del siglo $X$ que apenas conserva algún dato de interés para nuestro estudio (15). La segunda de ellas es de bastante más enjundia, la descripción geográfica de al-Andalus contenida en la primera parte de la Crónica de Ahmad al-Rāzì (16). La citada crónica histórica no se ha conservado en su

(10) AL-YACQUBI: Kitab al-buldan. B.G.A., t. VII, Ed. M.J. DE GOEJE, Leyden, 1892. Traducción francesa de G. WIET: Le pays. El Cairo, 1937. Los datos sobre al-Andalus traducidos en J. ALEMANY BOLUFER, op cit., pp. 115-119 y resumidos en G. CORNU, pp. 13-14. Sobre este autor, A. MIQUEL, op. cit., pp. 285 y sS

(11) IBN AL-FAQIH AL-HAMADANI: Kitab al-buldan. Ed. M.J. DE GOEJE, en B.G.A., t. V, Leyden, 1885. Trad española de la parte de al-Andalus en J. ALEMANY BOLUFER, op. cit., pp. 119-122. Sobre este autor, vid el artículo de H. MASSÉ en E./., III, pp. 784-785.

(12) AL-ISTAJRI: Kitab al-masalik wa-f-mamalik. Ed. M.J. DE GOEJE, B.G.A., t. I, Leyden, 1873. Trad. esp. de la parte referente a al-Andalus en J. ALEMANY BOLUFER, op. cit, pp. 123-137.

(13) IBN HAWQAL: Kitab surat al-And. Ed. M.J. DE GOEJE, B.G.A., t. II, Leycen, 1873. Existe otra edición de J.H. KRAMERS, Leyden, 1938 y otra reciente de 1979 que no hemos consultado. Trad. española de la parte referente a al-Andalus, en J. ALEMANY BOLUFER, op. cit., pp. 127-136. Traducción de la parte de al-Andalus y Norte de Átrica en IBN HAWQAL: Configuración del mundo (fragmentos alusivos al Magreb y España). Trad. de M. J. ROMANY, Valencia, 1971. Por su mayor facilidad ésta es la traducción que seguimos.

(14) AL-MUQADDASI: Kitab al-buldan. Ed. M.J. DE GOEJE, B.G.A., t. III, 2. ${ }^{a}$ ed. Leyden, 1906. Trad. francesa de la parte que nos interesa en Ch. PELLAT: Description de l'Occident musulman au IV $X$ siècle. Argel 1950. Traducción española de la parte de al-Andalus en J. ALEMANY BOLUFER, op. cit., pp. 143-152.

(15) AL-ZAYYĀT: Dikr al-Agalim wa-jitilatuha, obra que se conserva en manuscrito en la Biblioteca Nacional de París. Ha sido traducida parcialmente al español por F. CASTELLO: «Algunos capitulos del tratado de geografía árabe... de al-Zayyat». Estudios sobre historia de la ciencia árabe, Barcelona, 1980, pp. 115-151.

(16) Ediciones de la parte geográfica en P. DE GAYANGOS: Memoria sobre la autenticidad de la Crónica deno minada del moro Rasis. Madrid, 1850, pp. 33-63; D. CATALAN y M.S. DE ANDRÉS: Crónica del moro Rasis Madrid, 1975, pp. 11-118, y el estudio de E. LÉVI-PROVENÇAL: “La description de I'Espagne d'Ahmad 
original árabe sino en la traducción castellana medieval realizada, a su vez, de una previa traducción portuguesa. Pese a estos vericuetos de trasmisión, la parte geográfica está relativamente poco alterada y nos ofrece un panorama bastante desarrollado acerca de las producciones económicas de al-Andalus de donde se infieren datos sobre el comercio. La tercera fuente a la que nos referimos es una descripción anónima de al-Andalus recientemente publicada y traducida al español (17). Pese a conservarse en un manuscrito marroquí de comienzos del siglo XV, la mayor parte de sus datos geográficos reflejan la situación del siglo $X$.

Finalmente, estas fuentes geográficas, orientales y occidentales, de los siglos IX y $X$ pueden también completarse con otros autores posteriores. Básicamente nos referimos a dos de ellos que reflejan la situación de los siglos XI y XII, respectivamente. La primera de ellas es la detallada descripción geográfica de Abū 'Ubayd al-Bakrı. De ella se conserva íntegra la parte referida al Norte de África pero únicamente fragmentos de la de al-Andalus (18). La segunda obra es la del ceuti al-idrisi, probablemente el más importante geógrafo medieval (19).

Tanto en un caso como en el otro nos hablan de algunos artículos de comercio, así como de la relación entre puertos andalusís y africanos. Los utilizamos como elemento comparativo y de ampliación de la documentación, pero siempre teniendo en cuenta un hecho: la situación de los siglos XI y XII no tuvo por qué ser la misma de la de los siglos IX y X. Por ejemplo, cuando al-Idrisı documenta que en Játiva «se fabrica un papel que no tiene igual en todo el universo, se exporta a oriente y occidente» (20), confirma la continuidad del comercio ya existente en el siglo X. Pero cuando el mismo idrisi nos habla de la extraordinaria importancia del puerto de Denia, que «parten barcos que van hasta los rincones más lejanos de oriente, y de aquí es de donde parte la flota en tiempo de guerra» (21), está reflejando la situación del siglo XII pero no la del siglo X cuando ese mismo papel lo desarrollaba Almería.

\section{Los puertos de contacto}

En el sigio $X$ tanto al-Andalus como el Norte de África se caracterizaron por ser zonas de un intenso desarrollo comercial. Tanto en un caso como en el otro se trataban de regiones fronterizas, en contacto con pueblos extraños. Esta situación potenciaba la obtención y comercialización de productos de carácter «exótico» que, por sus características, tienden a ser más destacados por los escritores que los de tipo

al-Razì. Essai de reconstitution de l'original arabe et traduction française». Al-Andalus, 18, 1953, pp. 51-108. Igualmente puede verse, J. ALEMANY BOLUFER, op. cit., pp. 136-143.

(17) L. MOLINA: Una descripción anónima de al-Andalus. Ed. y trad., 2 tomos, Madrid, 1983.

(18) La parte referida al Norte de África, AL-BAKRI: Description de l'Afrique Septentrionale. Ed. y trad. de SLA. $\mathrm{NE}, 2{ }^{2}$ ed., París, 1965. Los fragmentos referidos al-Andalus han sido objeto de una edición crítica por $\mathrm{A}$ AL HAYYI, Beyrouth, 1968. Trad. española de E. VIDAL: Geografia de España (Kitab al-masalik wa-l-mamalik). Zaragoza, 1982. M. DE EPALZA, op. cit., pp. 26-28 ha utilizado el texto referente a contactos entre puertos norteafricanos y Sharq a!-Andalus.

(19) IDRIISSI: Description de l'Afrique et de l'Espagne. Ed. y trad de R. DOZY y M.J. DE GOEJE, París, 1866 reimpresión, Leyden, 1968. La traducción esp. de E. SAAVEDRA: La descripción de España del Edrisi. Madrid, 1881, es menos aconsejable; J. ALEMANY BOLUFER, pp. 154 y ss.

(20) IDRISI, p. 235 de la trad. francesa.

(21) IDRTSI, p. 234 
agrícola. Un intenso comercio marítimo de al-Andalus que sirve de fuerte contraste con la situación contemporánea de la Europa cristiana caracterizada por la atonía comercial (22).

Al-Andalus fue el centro difusor de elementos procedentes de los países cristianos. Ibn Jurdadbih nos documenta este trasiego comercial; según su testimonio una parte del mismo se hallaba básicamente desarrollado por mercaderes judios: «estos comerciantes hablan el árabe, el persa, el romano, las lenguas franca, española y eslavas. Viajan de Occidente a Oriente y de Oriente a Occidente, tanto por tierra como por mar. Del Occidente transportan eunucos, mujeres esclavas, muchachos, brocados, pieles de castor, pieles de marta y otras pieles, así como espadas» (23). El papel comercial de los judíos de al-Andalus no puede extrañarnos si tenemos en cuenta que, según al-Muqaddasi, en este país eran muy numerosos los elementos hebreos (24).

El comercio norteafricano también se caracterizaba por la procedencia foránea de muchos de los productos exportados al Oriente. Por ejemplo, del Sudán se obtenían grandes cantidades de esclavos negros y además volúmenes importantes de oro (25).

Los judíos también participaban en la extensión de este comercio por el Norte de África, tal y como documenta el mismo Ibn Jurdadbih: «Estos diferentes viajes se realizan igualmente por tierra. Los comerciantes que parten de al-Andalus o del país de los francos van hasta el Sus al-Adná; después desde Tánger se ponen en marcha hacia Ifriqiya.... (26). Aquí se detecta un viaje marítimo desde al-Andalus hasta un puerto del Norte del reino de Fez, sin duda el mismo de Tánger; después ya el itinerario se hacía por tierra. Un largo itinerario, puesto que al-Istajri consideraba que Fez distaba de Kairuán nada menos que 86 jornadas de camino. Según Ibn Jurdadbih, el Sous el Adna se hallaba a 2.150 millas de Kairuán. Más adelante, al-Bakri in-

(22) El libro clásico al respecto es el de H. PIRENNE: Mahoma y Carlomagno. Madrid, 3. a ed., 1981, pp. 207-208: "el intermediario ya no es el mar sino España. A través de ella los judíos están en relación con las potencias del África musulmana y con Bagdad». El autor destacó bien la atonia comercial de los países cristianos de Europa occidental, no obstante, su tesis acerca de que fue producto de la expansión islámica por el Mediterráneo está invalidada.

(23) IBN JURDADBIH, p. 114. Fl texto ha sido objeto de múltiples análisis y controversias. D. SIMONSEN: «Les marchands juifs appelés Radanites». Revue des Etudes Juives, 54, 1907, pp. 141-142; L. RABINOWITZ: Jewish merchant adventurers. Londres, 1948; B. BLUMENKRANZ: Juifs et chrétiens dans le monde occi dentale (430-1096). Paris-La Haya, 1960, pp. 13-14; E. ASHTOR: "Gli ebrei nei commercio mediterraneo nell'alto medioevo (sec. X-XI)». Gil Ebrei nell'Alto Medioevo. XXVI Settimane di studio del centro italiano di studi sull'alto medioevo, Spoleto, 1980, pp. 401 y ss.

(24) AL-MUQADDASI, p. 239. Más adelante volveremos sobre el papel de los judios. Acerca de los mismos, Cfr. E. ASHTOR: The Jews of Moslem Spain, t. I, Filadelfia, 1973. Sobre los judios en el Norte de Africa, H.Z. HIRSCHBERG: A History of the Jews in North Africa. $2{ }^{2}{ }^{2}$ ed., $t .1$, Leyden, 1974.

(25) Pueden verse los textos al respecto recogidos en J.M. CUOQ: Recueil des sources arabes concernant / Afri. que occidentale du VIII au XV/ siècle. París, 1975. El mejor texto es el del andalusi AL-ZUHRI: «Es de la región de Sus al-Aqsá de donde parten las exportaciones del Sáhara en servidores, esclavos... De allí se va a Tafilatet y Siyilmasa. Es de estos dos centros desde donde se dirigen los mercaderes de esclavos y todos los exportadores de esclavos, servidores, oro, marfil, ébano, defensas de elefantes, pieles de cierVOS...». AJ-ISTAJRI y MA'SUDI mencionan las grandes cantidades de oro exportadas desde Siyilmasa.

(26) IBN JURDADBIH, p. 116 (seguimos la traducción más correcta de M. HADJ SADOK. GOEJE incorrectamente transcribe Sous al-Aksá). El mismo texto en IBN FAQIH AL..HAMADANI confirma que estos comerciantes eran los judíos Radanitas. 
dicaría sobre Fez: «os judíos son más numerosos en Fez que en ninguna otra ciudad del Magrib; desde aquí hacen viajes a todas las partes del mundo» (27).

No obstante, los productos mencionados hacen referencia, sobre todo, a las exportaciones realizadas al Oriente islámico. La ruta comercial nos indica que no solamente nos debemos hallar ante elementos de aprovisionamiento de esos productos. En doble dirección, tanto al-Andalus debió de nutrirse de esos elementos procedentes del Sáhara (por ejemplo, esclavos negros), como ciudades del reino de Fez pudieron adquirir elementos ya mencionados procedentes de al-Andalus. De hecho, Ibn Judadbih, en otro párrafo, habla del comercio desarrollado (ya no por los judios) en el mar del Magreb, mencionando como productos los esclavos eslavos, romanos, francos y longobardos, las muchachas romanas y españolas, pieles de castor y otros animales, aromas, bálsamo de estoraque, drogas y el coral obtenido en las costas de Portugal (28). Son productos procedentes de al-Andalus pero que se comercializaban a través del Magreb. Geógrafos orientales, como Ibn Hawqal o alMuqaddasi, englobaban con el nombre de uproductos del Magreb» los exportados tanto por al-Andalus como por el Norte de África.

Lo que nos interesa en este trabajo es el comercio en las dos direcciones entre al-Andalus y el Norte de África. Los geógrafos orientales mencionados no dejan de indicar que se trataba de una zona caracterizada por el profuso desarrollo comercial. Uno de los geógrafos que hemos mencionado con anterioridad, al-MuqaddaSi, afirmaba que en el siglo $X$ tanto al-Andalus como el conjunto del Magreb destacaban en una determinada cuestión sobre el conjunto del mundo islámico: tanto en un lugar como en el otro abundaban especialmente los comerciantes y los viajeros (29). Ibn Hawqal destaca el esplendor comercial de al-Andalus, indicando el buen funcionamiento del mercado y de los precios (30). A mediados del siglo $X$, en el texto de una epístola, el judío Hasday ibn Shaprut, visir del Califa de Córdoba describía al-Andalus señalando: «Aquí llegan mercaderes de todos los confines de la tierra. Comerciantes de todo estado e isla confluyen aquí, desde Egipto y desde las otras naciones... El ingreso anual, que se me comunica a mí alcanza los cien mil dinares de oro. Éste es el promedio de sus ingresos anuales. Esto se debe a la cantidad de mercaderes que vienen de todas las tierras e islas. Todas las transacciones que con ellas se hacen están bajo mi control» (31).

Al-Andalus y el Norte de África eran vistos por los orientales como un conjunto lejano; otro autor oriental del siglo X, al-Masudi, indicaba que andalusís y mogrebies, pese a ser musulmanes, debían de ser considerados como auténticos extranjeros (32). En consecuencia, ese lugar lejano, repleto de comerciantes y viajeros, era ana-

(27) BAKRi, p. 262 de la Ed. y p. 226 de la trad. francesa.

(28) IBN JURDADBIH, p. 92 de la edic. y 66 de la trad.

(29) AL-MUQADDASI, p. 236.

(30) IBN HAWQAL, p. 66

(31) HASDAY IBN SHAPRUT: «Carta a los Jazares», trad. castellana de C. DEL VALLE: La Escuela Hebrea de Córdoba. Madrid, 1981, p. 337.

(32) Ch. PELLAT: «La España musulmana en las obras de al-Ma'sudi». Primer Congreso de Estudios Arabes e Islámicos, Madrid, 1964, p. 264. 
lizado como unidad, elemento que demuestra las profundas interconexiones económicas.

Para los geógrafos árabes orientales de los siglos IX y X, el concepto de Magreb no solamente abarcaba el Norte de África sino también al-Andalus. Significativa es la mención de al-Istajrī, consideraba que el Magreb se encontraba dividido en dos partes separadas por el Mediterráneo; en la orilla africana se encontraban Barqa, Ifriqiya, Tahert, Tánger y el Sus; en la orilla europea el Magreb incluía al-Andalus. El geógrafo oriental, que escribía en este punto siguiendo un mapa, señalaba que en al-Andalus las dos partes del Magreb llegaban a ponerse en contacto (33). AlMuqaddisi menciona Ceuta como una ciudad situada justo en el lugar donde ya se hacia visible la cercana costa de al-Andalus (34). Otros geógrafos árabes orientales de los siglos IX y $X$ también incluyen al-Andalus como una parte del Magreb (35).

En estas relaciones entre las costas de dos territorios vistos desde Oriente como unidad, hay que delimitar los principales puertos utilizados por el comercio hispanoafricano. Unas relaciones comerciales que seguian determinados itinerarios marítimos que, en no pocos casos, eran la continuidad de los existentes en la antigüedad clásica. $Y$ unos trayectos marítimos que, con ligeras variaciones, continuarían existiendo en siglos posteriores. Tanto al-Bakrī como al-Idrisis, incluso posteriormente Abulfeda e Ibn Sa'id al Magribi, documentan la existencia de puertos de contacto entre al-Andalus y el Norte de África.

Hay que tener en cuenta que, prescindiendo de las relaciones con países cristianos, todo el comercio de al-Andalus con el mundo árabe utilizaba el intermedio norteafricano. Al-Istajri podía indicar, con razón, que la ciudad de Túnez tenía el control sobre el paso hacia al-Andalus, sin su permiso no se podía pasar a la península (36). Este dato nos demuestra hasta qué punto los intereses comerciales se sobreponían a los político-religiosos.

Por otra parte, en esa comunicación marítima norteafricana, al-Andalus se encontraba menos alejada que otras tierras del mismo Norte de África. Es decir, que en ningún momento al-Andalus se vio al margen de los grandes circuitos comerciales del mundo islámico, muy al contrario, fue el eje principal del tráfico comercial existente en el Occidente (37).

Podemos poner un ejemplo en los itinerarios norteafricanos descritos por los propios geógrafos árabes, especialmente al-Istajrĭ, Ibn Hawqal y al-Muqaddisi. Según al-Istajñ desde Kairuán hasta Fez, capital del Estado de los idrisíes, se tardaban 86 jornadas de camino por tierra (38). Es cierto que esta cifra resulta algo exagerada

(33) AL-ISTAJRI, p. 36.

(34) AL-MUQADDASI, ค. 229.

(35) IBN AL-JURDADBIH, p. 89; AL-YACQÜBİ, p. 353; IBN HAWKAL, p. 41; AL-MUQADDAST, p. 215.

(36) AL-ISTAJRT, p. 38.

(37) No existe ningún estudio mínimamente completo acerca de las líneas de navegación en el Occidente islámi co. A falta de estudio tan necesario, pueden verse algunos datos en los trabajos de F. MORALES BE $L D A$ La marina de al-Andalus. Madrid, 1971; «La unidad de medida de los itinerarios maritimos en al-Andalus siglos VIII a XV». Actas de las / Jornadas de Cultura Árabe e Islámica, Madrid, 1981, pp. 165-177.

(38) AL-ISTAJRT, p. 46. 
por cuanto debe referirse a un trayecto realizado a pie; Ibn Jurdadbih reduce este trayecto a 54 días por realizarse en monturas (39). En todo caso, para ahorrar tiempo, los comerciantes podían utilizar un itinerario mixto, primero por mar desde Túnez a Tenes (5 ó 10 días), luego por tierra desde aquí hasta Fez (24 jornadas) (40). Este trayecto de Kairuán a Fez no podía realizarse en menos de una treintena larga de días. Por el contrario, como documenta al-Yáqūbì e lbn al-Jurdadbih, el paso desde Kairuán se hacia, primero en dos días a Túnez, seis (u once) hasta la costa de al-Andalus, y cinco jornadas más por tierra hasta Córdoba (41). Es decir, si el trayecto Fez-Kairuán se recorría entre 35 y 50 dias, el paso desde Kairuán a Córdoba se podia realizar entre 13 y 18 dias (42). Este análisis permite observar que las costas andalusís, incluso sus ciudades más importantes del interior, se hallaban menos alejadas de Kairuán y Túnez que las propias tierras de Marruecos.

A la hora de limitar los puertos comerciales, en la relación entre al-Andalus y el Norte de África, tenemos mucho mejor documentados los núcleos norteafricanos que los hispanos. Podemos hacer una breve relación, sin ánimo de exhaustividad, de los principales puertos hispanos que se encontraban en relación con el comercio africano. Siempre teniendo en cuenta que los productos con destino al Oriente islámico seguían los mismos derroteros.

Tenemos, en primer lugar, comenzando por el Mediterráneo, el puerto de Tortosa. Esta ciudad en el siglo IX ya se había convertido en la puerta de al-Andalus. Su importancia vino determinada por ser la salida al mar de los productos comerciales generados en la cuenca de Ebro, región de Zaragoza, y de los procedentes de la tierra de los francos. Ahmad al-Rāzì nos la describe señalando que «Tortosa ha muy buen termino e buen puerto, e los mercadores vienen y de toda la parte de la tierra, e es çerca de los de Françia» (43).

Un segundo puerto comercial era el de Valencia. Con el tiempo, esta ciudad fue especializando su comercio como dirigido hacia el Oriente del Mediterráneo. Para la época que nos interesa tenemos la mención concreta de Ahmad al-Rāzī acerca de la exportación de un producto concreto: «e a tanto açafran que abondaria a toda España, e dende lo lievan los mercadores a todas las partes del mundo" (44). Al Sur de Valencia, Ahmad al-Rāzi menciona «un puerto a que llamavan Donea es muy bueno e muy antiguo» (45). Más hacia el Sur destacaba el puerto de Cartagena, también mencionado por Ahmad al-Rāzi. Era el puerto de la Kora de Tudmir. El geógrafo al-Ya'qūbı describe al-Andalus a partir de la narración de un viajero pasado

(39) IBN JURDADBIH, pp. 87-88. Cifra mucho más acertada. La distancia de 54 días entre Kairuán y Fez es también la que dan IBN AL-FAQIH AL-HAMADAN1, pp. 79 y 81, y QUDÄMA: Kitāb al-Haräğ wa Sinärat al-Kitaba. Ed. y trad. de M.J. DE GOEJE, B.G.A., VI, Leyden, 1889, p. 207.

(40) Cfr. el repertorio de caminos norteafricanos de IBN HAWKAL. La misma conclusión puede obtenerse si tenemos en cuenta los itinerarios norteafricanos mencionados por AL-MUQADDAST, pp. 243 y ss. El mismo BAKRT, p. 315 (p. 271 de la trad. francesa), señalaba que de Fez a Kairuán habia 40 jornadas de marcha.

(41) AL-YA'QÜBÏ, p. 353; IBN JURDADBIH, p. 87

(42) AL-ISTAJRT̄, p. 40, destacaba que Córdoba, en al-Andalus, y Kairuán, en Ifriqiya, eran las ciudades más majestuosas, grandes y ricas de todo el Magrib. Era lógica una fácil comunicación entre las dos.

(43) AHMAD AL-RAZI, cap. VIII; Ed. D. CATALÁN y M.S. DE ANDRÉS, p. 39.

(44) AHMAD AL-RĀZIT, cap. VII; Ed. mencionada, p. 38.

(45) AHMAD AL-RĀZII cap. Vl; Ed. mencionada, p. 36. 
desde el Norte de África. El mismo indica que, dejando la costa norteafricana, «se dirige entonces a la península de al-Andalus cortando por alta mar durante un día y una noche, hasta que llega al país de Tudimir, una región amplia y habitada en la que hay dos ciudades llamadas Al-Askar y Lorca, ambas con mezquita mayor» (46). El puerto de desembarco, junto a Al-Askar, en ruta a Lorca y Elvira, no puede ser otro que el de Cartagena (con mucha mayor probabilidad que Águilas).

Ésta es la única descripción detallada que tenemos para el siglo IX de la navegación entre el Norte de África y al-Andalus. Nos hallamos con un paso realizado con anterioridad a la fundación de Pechina, igualmente cuando el paso Ceuta-Algeciras todavía no estaba del todo desarrollado. Nos podemos preguntar si nos hallamos ante una excepción o podemos conceder categoría de generalidad al trayecto documentado por al-Yaqubi. No es este el único caso atestiguado de pase desde el Norte de África directamente al puerto de la Kora de Tudmir (47). En realidad, son muy pocos los pasos atestiguados en los siglos VIII y IX. Simplemente puede concluirse que, con toda probabilidad, el pase desde las costas argelinas a las de Sharq al-Andalus fue comparativamente mucho más corriente en el siglo IX que en el $X$.

Pechina y su arrabal marítimo de Almeria se convirtió en el siglo $X$ en el principal puerto comercial de al-Andalus, en la salida al mar de Córdoba. El hecho está suficientemente documentado en la obra de Lévi-Provençal, por lo que nos excusamos ahora de mayores consideraciones. Volveremos más adelante sobre el mismo a la hora de tratar de los puertos norteafricanos.

Málaga y Algeciras fueron, igualmente, dos puertos relacionados ampliamente con el comercio norteafricano. El primero de ellos no tuvo gran importancia en los momentos que tratamos, su desarrollo comercial es sobre todo posterior. Algeciras, por el contrario, se fue convirtiendo en un puerto de trasiego considerable en relación con la cercana ciudad africana de Ceuta. Esta consideración se refiere particularmente al período del siglo $X$ cuando la conquista Omeya de Ceuta fortaleció las relaciones entre ambas ciudades consideradas como hermanas (48). Ahmad al-Räzĭ la menciona indicando que «su puerto es muy çercano para pasar allende la mar, e paresçe denbe Çebta, que es villa en que ha mucho bien» (49).

Finalmente, Sevilla tiene que mencionarse como un puerto fluvial de gran trasiego comercial. Ahmad al-Rāzì la menciona en dos ocasiones como exportadora. En primer lugar, del aceite, «dende navios contra el sol levante e para todas las partes del mundo; e tanto es que sy non levasen, non lo guardarian ni darian por ello ninguna cosa» (50). En segundo lugar, Rāzi indicaba que Sevilla «en su termino ha mucho algodon e dende lo lievan para tierra de ultramar e a la de promiçion» (51). Al-'Udrī indicará que el algodón de Sevilla se exportaba a Ifriqiya.

(46) AL-YAQUBI, p. 353

(47) Por ejemplo, IBN CIDART: Al-Bayan al-Magrib. Trad. francesa de E. FAGNAN, t. II, Argel, 1901, p. 88.

(48) IBN HAYYAN: Al-Muqtabis, V. Ed. de P. CHALMETA, F. CORRIENTE y M. SUBH, Madrid, 1979, p. 191; trad. de M.J. VIGUERA y F. CORRIENTE, Zaragoza, 1981, p. 217: «ciudad de Ceuta, en la costa africana, puerto de paso desde ésta a al-Andalus frente a Algeciras, puerto de paso de al-Andalus a la costa africana».

(49) AHMAD AL-RÄZİ, cap. XXXV; Ed. mencionada, p. 104

(50) AHMAD AL-RÄZZT, cap. XXXI; Ed. mencionada, p. 92.

(51) AHMAD AL-RÄZZI, cap. XXXI; Ed. mencionada, p. 93. 
Los puertos norteafricanos que practicaban el comercio con andalusís están mejor documentados en las descripciones de los geógrafos árabes. En la zona de Ifriqiya el comercio con al-Andalus estaba muy desarrollado. Ibn Hawqal menciona las tasas cobradas en los puertos cercanos sobre el comercio con Bizancio y con al-Andalus, como la principal fuente de riqueza de Kairuán (52).

lbn Jurdadbih indica sobre Túnez: «esta ciudad se encuentra situada a dos días de marcha en mula de Kairuán. Está situada al borde del mar... Túnez está separada de al-Andalus por el mar que en este lugar tiene 6 parasangas de largo. Después de hacer este trayecto se va a Córdoba, la capital de al-Andalus, en cinco jornadas» (53). Exactamente el mismo texto encontramos recogido en Ibn al Faqin al-Hamadāni (54). Nos hallamos ante un evidente error de lbn Jurdadbih, lapsus seguido por Ibn al-Fagîh. Con toda probabilidad el geógrafo persa había querido escribir que la navegación desde el puerto de Túnez hasta al-Andalus era de seis jornadas, como demuestra la referencia posterior a cinco jornadas como distancia de Córdoba a la costa, pero se le deslizó en lugar de «jornadas» el nombre de la medida persa.

En el año 921 al-stajī nos describe la ciudad de Túnez demostrando que continuaba teniendo una enorme importancia en la navegación y comercio hacia alAndalus: «La ciudad de Túnez dista dos días de Kairuán. Túnez es una ciudad grande y extensa, con un territorio amplio y fertil, rico en aguas y sembrados. Es el primer punto de paso hacia al-Andalus; a ésta no se puede pasar sin su control» (55). Los textos recogidos nos indican que en el siglo IX y primer tercio del siglo $\mathrm{X}$, el puerto de Túnez controlaba un intensísimo comercio marítimo con al-Andalus.

La situación cambió ostensiblemente en los años anteriores a mediados del siglo X. Túnez dejó de ser el gran puerto de control del comercio andalusí. En la descripción de Ibn Hawqal, que visitó el lugar hacia el 947, la economía tunecina era ya básicamente agrícola, no mencionando el comercio como fuente de riqueza (56). Túnez había sido sustituido ya en esas fechas por Mahdiya, ciudad fundada muy pocos años antes. Cuando la visitó Ibn Hawqal estaba en pleno esplendor; era el puerto de Kairuán y allí se practicaba muchísimo comercio (57). Por al-Bakrī y alIdrisi sabemos que todavía en los siglos XI y XII el puerto de Mahdiya era uno de los principales a los que accedían comerciantes andalusís.

No obstante, a mediados del siglo $X$ el puerto más importante del comercio entre al-Andalus e lfriqiya era el de Tabarqa. Al-istajri todavía la cita como una ciudad pequeña, no destacando para nada un desarrollo comercial (58). En los años siguientes, Tabarqa se convirtió en un puerto que practicó un intenso comercio con al-Andalus tal y como indica Ibn Hawqal: «En cuanto a la ciudad propiamente dicha de Tabarqa, a pesar de su pequeña superficie y su condición modesta, ha llegado a ser céle-

(52) IBN HAWKAL, p. 49 de la trad. de M.J. ROMANI.

(53) IBN JURDADBIH, p. 87 del texto, y 63 de la trad. francesa.

(54) IBN AL-FAQITH AL-HAMADĀNI, p. 79.

(55) AL-ISTAJRT, p. 38.

(56) IBN HAWQAL, p. 24

(57) IBN HAWQAL, p. 21

(58) AL-ISTAJRI, p. 38. 
bre por la gran cantidad de navíos que allí anclan, en especial negociantes andalusís que descendían a este puerto y sobre los que se establecia en otra época un diezmo. Por la situación que ocupa, está situada frente a las orillas más próximas de alAndalus» (59).

Otro puerto importante en relación con al-Andalus es el de Bona que con el tiempo se fue imponiendo poco a poco al de Tabarqa. Bakri documenta el comercio andalusí en este puerto (60). Ibn Hawkal habla de la exportación de sus mercancias, aunque no cite expresamente la llegada de comerciantes andalusís: exportaba cereales, hierro, lana y, sobre todo, ganado y bestias de carga (61).

La costa argelina también se caracterizó por un intenso trasiego comercial con al-Andalus. Argel tal y como la describen al-Istajī e Ibn Hawqal no parece que fuera un gran puerto comercial; en todo caso, su zona más próxima destacaba por la existencia de grandes rebaños de carneros y. bueyes (62). Sin embargo, por al-Bākri sabemos que este puerto mantuvo considerables relaciones con al-Andalus (63). Lo menciona como situado frente a Peñíscola, de la que distaba una navegación de 6 dias.

Mención especial tiene que hacerse de Tenes. Al-Ya'qūbī la menciona como un puerto ubicado a cuatro dias de Tahert, situado frente a al-Andalus y que era el lugar de paso hacia ella (64). En realidad, Tenes habia sido una fundación realizada por los andalusís en el año 875: «Los marinos de los que hemos hablado tenian por costumbre el dejar al-Andalus y pasar el invierno en el puerto de Tenes. Los bereberes de los alrededores, que se reunían con ellos, les invitaron a establecerse en la fortaleza y a situar un mercado, prometiendo proveerles, favorecerles y observar hacia ellos las obligaciones de amistad y buena vecindad" (65). Al menos una parte de estos marinos, emigrados nuevamente después desde Tenes, iban a ser los fundadores de Pechina (66).

Al-Istajrit también documenta Tenes como lugar de relación con al-Andalus al indicar que se hallaba frente a ella. Ibn Hawqal documenta el intenso comercio andalusí: «es la ciudad a la que llega una mayor cantidad de navíos de los andalusís, donde traen sus mercancías y donde las embarcan hacia otros lugares» (67).

El puerto de Orán también era en el siglo $X$ frecuentado por los comerciantes andalusís. Ibn Hawqal afirma que Orán «es un puerto de comercio donde los españoles traen mercancias y donde cargan cereales» (68). De hecho, también la ciudad de Orán misma había sido fundada en el año 902 por andalusís (69). A lo largo del

\footnotetext{
(59) IBN HAWQAL, p. 25

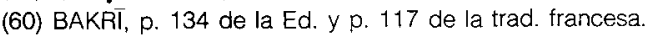

(61) IBN HAWKAL, p. 26.

(62) AL-ISTAJPIT, p. 38; IBN HAWQAL, p. 27.

(63) BAKRT, p. 157 de la Ed. y p. 137 de la trad.

(64) AL-YA'QUBT, p. 358.

(65) BAKRí, p. 147 de la Ed. y p. 128 de la trad.

(66) BAKRT, p. 148 de la Ed. y p. 129 de la trad.

(67) AL-ISTAJRT, p. 38; IBN HAWQAL, p. 28.

(68) IBN HAWQAL, p. 28

(69) BAKRं, pp. 166-167 de la Ed. y pp. 144-145 de la trad.
} 
siglo $X$ Orán se convirtió en un puerto esencial en el comercio andalusí con el Norte de África, superando incluso al de Tenes. Al-Muqaddisi indicaría que la travesía de Orán hacia al-Andalus se hacía de forma constante tanto de día como de noche (70). Esta mención nos indica que era el principal puerto de tránsito tanto para hombres como para mercancías.

La situación perduró mucho en el tiempo; en el siglo XII al-Idrīsí indicaría que era de Orán de donde tomaban los comerciantes de al-Andalus la mayor parte de sus aprovisionamientos agrícolas, «os navíos andalusís llegan sin interrupción a sus puertos» (71). Al-Bakri sitúa Orán frente a Escombreras, de la que estaba separada por una navegación de dos días y medio. Ello quiere decir que la navegación hacia el puerto de Orán se realizaba, sobre todo, desde Cartagena y desde Almería.

A partir de ese punto comenzaba el espacio marítimo más cercano entre las dos orillas del Magrib. La navegación entre los puertos de una y otra costa apenas duraba en ocasiones una jornada; incluso en la zona del Estrecho se pasaba de Algeciras a Ceuta en unas pocas horas. El geógrafo al-BakrT nos informa de los puertos de contacto y de la longitud de la travesía. La travesía desde Ceuta a Algeciras se realizaba en apenas medio día (72). De Mezemma (Al-Hoceima) a Málaga se empleaba dia y medio de navegación, al igual que de Jete al cabo Tres Forcas (73). Desde Melilla hasta Almuñécar o Salobreña se empleaban dos días (74). Itinerarios marítimos del siglo XI que, indudablemente, continúan otros existentes en época califal (75).

La lista de puertos del actua! Marruecos, relacionados con al-Andalus en el período Omeya, se inicia con el de Melilla. De hecho, la ciudad llegaría a ser conquistada por los Omeyas para consolidar una cabeza de puente en la zona, momento en el cual alcanzó su máximo desarrollo económico (76). No obstante, en general, el trasiego comercial de Melilla no pasó de un volumen modesto: algunos productos agrícolas obtenidos por comerciantes andalusis (77). No obstante, de la referencia de Ibn Hawqal se deduce que la mayor parte de sus producciones servían meramente para el autoconsumo (78).

Mayor importancia habia adquirido con anterioridad a esas fechas el comercio hispano con el reino de Nakur. Dicho reino mantuvo siempre relaciones de sometimiento con los Omeyas de al-Andalus; en el siglo X, las tropas Omeyas tomaron buena parte del reino ante la presión ejercida por los fatimidas. La capital del rẹino se

(70) AL-MUQADDASI, p. 229.

(71) IDRIST, pp. 96-97.

(72) BAKRI, p. 239 de la Ed. y p. 206 de la trad.

(73) BAKRI, p. 227 de la Ed. y 197 de la trad.

(74) BAKRT, p. 228 de la Ed. y 197 de la trad.

(75) Con la matización de que en el siglo XI ya no aparece mencionada la navegación norteafricana hacia Pechina. En el siglo XII, al-Idrisi muestra ya un cierto cambio en los itinerarios, aunque siempre en la navegación se indican jornadas de uno o dos días.

(76) E. GOZALBES: «Melilia, ciudad musulmana». Actas del Primer Congreso Hispano-Africano de las culturas mediterráneas. Granada, 1987, pp. 175-185.

(77) E. GOZALBES: «Melilla en el siglo XI: datos para su historia». Actas de las / Jornadas de Cultura Árabe e Islámica, Madrid, 1981, pp. 237-245.

(78) IBN HAWQAL, p. 29. 
hallaba en el valle de al-Hoceima y tenía su puerto principal en al-Mezemma. Nakur era una ciudad importante, en la cual en los siglos IX y $X$ habia muchos comerciantes (79). Se relacionaba fundamentalmente con los puertos andalusís de Málaga y Pechina. El recuerdo a estas relaciones comerciales de Nakur con Pechina llegaría a ser reflejado, siglos después, por Ibn al-Jatib (80). Hay que tener en cuenta que poetas del șiglo $X$ ya destacaban que el avituallamiento de Almería dependía del viento que trajera a mayor o menor velocidad las naves desde el Norte de África; al-Maqqa$\eta$ indicaría que era de la costa de enfrente, de Marruecos, de donde Almería hacía venir sus alimentos (81).

Al-Yaqubi describió elogiosamente la riqueza del reino de Nakur que consistía sobre todo en productos agrícolas y ganaderos. Afirmó con cierto error que Abdarrahman I embarcó aquí con destino a Almuñécar (82), lo cual puede ser un reflejo de esos múltiples contactos con la orilla andaluza. Al-Istajri, que primero la cita como uno de los puertos principales en la navegación norteafricana (83), después la menciona con elogio de la fertilidad de su territorio; nos indica otro dato importante al referir su conexión con Pechina (84).

En la península N.O. de Marruecos existían dos ciudades portuarias que mantuvieron un fuerte nivel de contactos comerciales con al-Andalus. Fueron estas ciudades las de Ceuta y Tánger. Con respecto a sus territorios dependientes, la península N.O. de Marruecos, la Córdoba Omeya estuvo en constantes relaciones tanto políticas como económicas. Este hecho llegó al culmen en el siglo X. Ibn Hawqal destacaba que estos territorios estaban separados de al-Andalus únicamente por un estrecho sobre cuyo carácter angosto se permitió exagerar: «la distancia entre las dos orillas es reducida hasta el punto de que se puede ver sobre el litoral opuesto, las gentes que caminan, la silueta de los árboles, la naturaleza de los cultivos».

Como vimos con anterioridad, hasta finales del siglo IX predominó la relación entre el Sharq al-Andalus y la costa argelina; fue en esa vía en la que se realizó una parte del comercio hispano-africano. El desarrollo de Pechina, convertido en principal puerto comercial de Córdoba, después la conquista de Ceuta por Abdarrahman III, desplazaron el eje de las relaciones.

Hasta esta conquista nada indica que Ceuta mantuviera considerables relaciones comerciales con al-Andalus. No pasaba de ser una modesta población portuaria. Con ese carácter modesto es mencionado por Ibn Jurdadbih e Ibn Faqih al-Hammadāni (85), mientras al-Yáqübī no la cita por su nombre; sin embargo, indica que era en su zona (sin duda para embarcar en la misma ciudad) donde se reunían los pere-

(79) IBN HAYYAN: Al-Muqtabis V, p. 281

(80) IBN AL-JATIB: Kitab A'mal al A'lam. Trad. de R. CASTRILLO, Madrid, 1983, p. 111.

(81) H. PÉRĖS: Esplendor de al-Andalus. La poesía andaluza en árabe clásico en el siglo XI. Madrid, 1983, pp. 149-150. Sin embargo, en un momento de crisis militar en Nakur, año 935, está documentado el aprovisionamiento de la misma desde Pechina, IBN HAYYAN: A/-Muqtabis V, p. 252.

(82) AL-YACQŪBT, p. 357

(83) AL-ISTAJRT, p. 37

(84) AL-ISTA.JRI, p. 38

(85) IBN JURDADBIH, p. 88; IBN FAQTHH AL-HAMMADÄNI, p. 79. 
grinos de las tierras de Marruecos (86). Más significativo es todavía el silencio de al-Istajir que indica que Ceuta no tuvo mayor importancia con anterioridad a la conquista Omeya. Sin embargo, las relaciones comerciales y humanas con al-Andalus debieron de ser bastante intensas. Así sabemos que hacia mediados del siglo IX se instaló en la ciudad una masa considerable de población procedente de Calsana (zona de Cádiz) (87).

Después de la conquista Omeya, Ceuta se convirtió en un puerto comercial de gran trasiego, asi como un lugar de salida y llegada de los viajeros. En consecuencia, se convirtió para las tierras y costas marroquies en lo que Orán era para las argelinas. Al-Muqaddasi describe Ceuta como el lugar desde el cual era visible al-Andalus, indicando que constituía un paso célebre hacia la misma (88). Ahmad al-Rāzī la cita como importante ciudad que servía de puerto de paso en relación con Algeciras (89).

Otro puerto importante fue el de Tánger. Muy probablemente el mismo alcanzó mayor importancia, en relación con el comercio hispano, en los siglos VIII y IX. La conquista de Ceuta por los Omeyas, en tiempos de Abderrahman III, desvió a Ceuta el principal lugar de paso. Las fuentes árabes son enormemente imprecisas a la hora de hablarnos de Tánger. Este hecho se produce porque en muchas ocasiones Tánger es utilizado no como nombre de ciudad sino como de un territorio, nombre de herencia de la antigua Mauritania Tingitana de los romanos. Éste es indudablemente el sentido que tiene el nombre de Tánger en lbn Jurdadbih. Además este autor menciona entre las ciudades principales una llamada al-Khadrâ ( «la Verde») que, según el geógrafo oriental, «se encuentra sobre el borde del mar que, en este lugar, no tiene nada menos que 6 parasangas de anchura" (90).

Al-Khadrâ era también el nombre de Algeciras ( = isla Verde). Pero en este caso ni de cerca ni de lejos puede referirse a la población andalusí, el texto es bien claro en lo referente a que la ciudad se hallaba en el reino de Fez. Además, Ibn Faqih al-Hammadāni menciona dos veces la ciudad como situada en el reino de Fez, cerca de Ceuta (91); al-Muqaddasi también la nombra en la misma zona (92). Resulta indudable que en la costa marroquí, cercana a Ceuta, existía una ciudad de cierta importancia que era nombrada como al-Khadrâ.

Una ciudad importante, a la orilla del mar, situada en la zona angosta del Estrecho, descartando Ceuta ya mencionada por el mismo geógrafo Ibn Jurdadbih, no puede ser otra que Tánger. La conclusión se confirma si tenemos en cuenta que lbn Jurdadbih menciona Ceuta como «situada al lado de al-Khadrâ» (93). Por otra parte,

(86) AL-YA'QUBI, p. 357

(87) AL-BAKRT, p. 237 de la Ed. y 204-205 de la trad.

(88) AL-MUQADDASI, p. 224.

(89) Cfr. nota 49.

(90) IBN JURDADBIH, p. 89 de la Ed. y 64 de la trad.

(91) IBN AL-FAQİH AL-HAMMADĀNI, p. 80

(92) AL-MUQADDAST, p. 221.

(93) IBN JURDADBIH, p. 88 de la Ed. y 63 de la trad. El paso desde Alcazarseguer hasta Tarifa iba a tener una gran importancia ya en época almorávide y almohade pero en la época Omeya no era utilizado con frecuencia, alguna mención al mismo hay en la segunda mitad del siglo X, y nunca por parte de los comerciantes. 
Ibn al-Qutiyya menciona Tánger, que se llamaba «La Verde». La referencia al «borde del mar» y a lo angosto del mar, nos indica que en esas fechas, primera mitad del siglo IX, Tánger era el principal lugar de paso hacia al-Andalus. Fue la conquista Omeya de Ceuta la que desvió el principal de los pasos de viajeros y de comerciantes hacia esta última ciudad. En la segunda mitad del siglo $X$ volvería Tánger a destacar como lugar de paso, si bien en este caso no está documentado con respecto al comercio sino al trasiego de tropas (94).

Pero el puerto de Tánger presentaba un problema en relación con el volumen comercial. Es bien sabido que en la antigüedad clásica esta ciudad, la Tingi romana, era el principal puerto de paso de viajeros y mercancias en relación con Hispania (95). Pero en la época Omeya el puerto fue frecuentado únicameitte por los barcos comerciales de pequeño tamaño: «este lugar es frecuentado por los navíos de pequeñas dimensiones que vienen a descargar sus mercancías, pero los grandes navíos no acceden porque es muy peligroso cuando el viento sopla del Este» (96).

Otro puerto de intenso contacto comercial con al-Andalus fue el de Arcila. Los andalusís habian mantenido frecuentes intercambios con la zona de Arcila que ya en el siglo VIII aparece en ocasiones como aprovisionadora de alimentos para la Península. La misma fundación de Arcila como núcleo urbano y portuario bien definido fue debida precisamente a la actuación de comerciantes hispanos. Al-Bakri nos documenta los episodios de esa fundación, subsiguientes a una presencia de normandos en el año 844. Según al-Bakri, debido a la riqueza cerealística y ganadera de los campos cercanos a Arcila, los comerciantes de al-Andalus comenzaron a establecer sus tiendas con diversas mercancias: «entonces se comenzaron a construir casas, una tras otra, terminando por formarse una ciudad» (97).

En la segunda mitad del siglo IX y en la primera del siglo $X$, el puerto de Arcila se convirtió en uno de los puntos fundamentales del comercio norteafricano de alAndalus. En el primer cuarto del siglo X, la ciudad sería mencionada con ese carácter por parte de al-Istajri: «Arcila es una ciudad grande a la orilla del mar Océano y es muy fertil y rica. Es una ciudad de paso hacia al-Andalus» (98). Este carácter portuario y la pujanza del comercio andalusi explica que fuera, junto con Melilla, Ceuta y Tánger, uno de los principales puntos sometidos y utilizados por los Omeyas en su expansión norteafricana. En la segunda mitad del siglo X será nombrada por Ibn

(94) ISA AL-RĀZī; trad. de E. GARCíA LÓPEZ: Anales palatinos del Califa de Córdoba al-tiakam Il. Madrid, 1967. Acerca del desarrollo de todas estas ciudades puede verse la recopilación de trabajos de G. GOZALBES BUSTO: Estudios sobre Marruecos en la Edad Media. Granada, 1989.

(95) La travesía corriente de hombres y mercancias se hacía desde Baelo Claudia (Bolonia) a Tingi, tal y como documentan STRABON III, 1, 8; PLINIO: N.H. V, 3; ttin. Marit., 495, 4-6. Sobre los itinerarios navales de comercio hispano-africano en la antigüedad, Cfr. E. GOZALBES: «Relaciones comerciales entre Carthago Nova y Mauritania durante el Principado de Auguston. Anales de la Universidad de Murcia, 40, 3-4, 1982, pp. 13-26; IDEM: «Carteia y la región de Ceuta. Contribución al estudio de las relaciones entre ambas orillas del Estrecho en la antigüedad clásica». Actas / Congreso internacional El Estrecho de Gibraltar, Madrid, 1988, pp. 1.047-1.067.

(96) AL-BAKRT, p. 248 de la Ed. y 214 de la trad.

(97) AL-BAKRT, p. 255 de la Ed. y 220 de la trad.

(98) AL-ISTAJRT, p. 39. El mismo AL-ISTAJRIT, pp. 41 y 45, repite por dos veces el dato erróneo de que Abderrahman I pasó a al-Andalus desde el puerto de Arcila. Es un nuevo indicio de la importancia que Istajr daba a Arcila como lugar de paso entre el Norte de África y al-Andalus. 
Hawqal, quien no hace referencia expresa al comercio andalusi, aunque destaca aquí la existencia de mercados importantes (99).

Hacia el Sur, la ciudad de Basra era otra que mantenia muy considerables relaciones comerciales con al-Andalus. No se trataba de una ciudad marítima sino interior, pero se hallaba comunicada con el mar. Era el centro de una gran zona agrícola, con otras ciudades menores dependientes (100). Ibn Hawqal nos informa que los comerciantes de Basra transportaban sus mercancías por navíos en el río, alcanzaban el Océano y desde allí se comunicaban con otros puertos atlánticos y mediterráneos (101).

Es al-Istajrī quien nos ofrece, hacia el año 921, un indicio acerca de la importancia del comercio andalusí con esta población marroquí. En primer lugar, la nombra como puerto de correspondencia africano, junto con Nakur y Arcila (102). Más adelante la cita como una «ciudad grande, extensa y de territorio fertil», poniéndola en relaciones con la que considera próxima zona andalusí de Gibraltar, aunque se equivoca al dar una travesía muy corta desde Basra (se entiende que su zona costera) y al-Andalus (103).

Pero es indudablemente lbn Hawqal quien nos documenta, mejor que nadie, la naturaleza de ese comercio andalusí practicado por Basra. Como la misma arqueología ha demostrado, la ciudad poseía un puerto en la cercana laguna de Moulay Bou Selham. El geógrafo oriental nombra esta laguna como lago de Aryag y dice sobre el mismo: «El lago de Aryag comunica con el Océano, donde anclan las naves andalusís que vienen a cargar los productos agrícolas de la región» (104). Y de Basra indica que era una ciudad repleta de opulentos comerciantes. Desde los inicios del siglo X Basra poseía una considerable colonia de andalusís (105). Significativamente, la ciudad también sería controlada militarmente por los Omeyas en época del Califa al-Hakam II (106).

Los puertos situados más hacia el Sur fueron objeto del comercio andalusí en fechas posteriores. Concretamente al-Idrīsi nos documenta que en el siglo XII, los barcos andalusís accedian a Salé, Fedala y Anfa para obtener productos agrícolas y ganado. Sin embargo, no tenemos atestiguado el acceso a estos puertos en los siglos IX y X cuando las condiciones políticas de esta zona, dominada entonces por la tribu beréber de los Bargawata, fueron notablemente diferentes. Ello hace supo- ner que el comercio hispano no se inició en la zona hasta la época almorávide.

Este dato supone que el comercio andalusí con ciudades del interior de Marruecos se realizaba a partir de los puertos del N.O. del territorio, los nombrados más

(99) IBN HAWQAL, p. 30 de la trad. mencionada.

(100) Sobre ella continúa siendo válido el trabajo que le dedicara D. EUSTACHE: «El-Basra, capitale idrissite, et son port». Hesperis, 42, 1955 , pp. 217-238.

(101) IBN HAWQAL, p. 31

(102) AL-ISTAJRT, p. 37

(103) AL-ISTA.JRI, pp. 38-39.

(104) IBN HAWQAL, pp. 32-33.

(105) AL-BAKRT, p. 113 de la Ed. y 221 de la trad.

(106) IBN 'IDARII, II, p. 402. 
arriba. Sabemos que los comerciantes andalusís accedían con mucha frecuencia a Fez, la capital del reino de los idrisíes. Al Sur de Fez se hallaba el importante núcleo de Siyilmassa. Ibn Hawqal, que visitó esta ciudad, la documenta como en pleno período de esplendor. A Siyilmassa accedían con cierta frecuencia comerciantes andalusís que llegaban en caravanas procedentes de Fez. Ibn Hawqal nos habla de los pingües beneficios obtenidos por el gobierno de la ciudad gracias al impuesto sobre las mercancías que llegaban o que partían de/hacia lfriqiya, Fez, el Sus, alAndalus o Agmat (107). Sin embargo, los comerciantes hispanos no se establecieron de forma definitiva en la ciudad sino que la utilizaron como lugar de acceso extremo en sus actividades. Así se deduce de otra cita del mismo geógrafo oriental: «las caravanas pasaban del Magrib a Siyilmassa, donde se establecieron habitantes de Iraq, negociantes de Basora y de Kufa, así como de Bagdad..., ellos y sus hijos llevaban sus mercancías, o viajaban sin bagajes; y sus ininterrumpidas caravanas cosechaban beneficios considerables, ganancias inmensas y provechos apreciables" (108).

Como hemos indicado, toda la relación comercial con el interior y sur de Marruecos se realizaba, en los siglos IX y X, a partir de los puertos situados en el N.O. del territorio. Este dato lo podemos ver confirmado por Ibn al-Jurdadbih cuando indicaba que en la costa sur de Marruecos no existía navegación (109). Y también se confirma en Ibn Hawqal que menciona la población de Salé como extrema y como una posición no comercial sino militar, de guerra santa (110).

(107) IBN HAWQAL, pp. 52-53.

(108) IBN HAWQAL, p. 10.

(109) IBN JURDADBIH, p. 93

(110) IBN HAWQAL, p. 33. 